\title{
Depressive symptoms as a mediator between impulsiveness and suicidal ideation among Chinese medical college students
}

\author{
Jingxuan Zhang \\ Army Medical University \\ Xiaolin Zhang \\ Army Medical University \\ Guoyu Yang \\ Army Medical University \\ Zhengzhi Feng ( $\nabla$ fzz@tmmu.edu.cn ) \\ Army Medical University
}

\section{Research Article}

Keywords: depressive symptoms, impulsiveness, suicidal ideation, mediating effect, cross-sectional analysis, longitudinal analysis

Posted Date: April 15th, 2021

DOI: https://doi.org/10.21203/rs.3.rs-398303/v1

License: (c) (i) This work is licensed under a Creative Commons Attribution 4.0 International License. Read Full License 


\section{Abstract}

Background: Suicidal ideation is thought to be of high risk for attempts and behaviours. Researches have suggested that depressive symptoms and impulsiveness can affect suicidal ideation, attempts and behaviours independently. But how they work together in influencing suicidal ideation remains unclear. This study is aiming to investigate the reciprocal associations among impulsiveness, depressive symptoms, and suicidal ideation and to confirm the mediating effect of depressive symptoms on impulsiveness and suicidal ideation through cross-sectional and longitudinal analyses.

Methods: The Self-rating Depression Scale (SDS), Barratt Impulsiveness Scale $11^{\text {th }}$ version (BIS-11) and Self-rating Idea of Suicide Scale (SIOSS) were applied, as well as a list of demographic information. Multilinear regression and parallel process latent growth curve models were applied to conduct cross-sectional and longitudinal analyses, respectively.

Results: (1) Participants with suicidal ideation scored higher in depressive symptoms and impulsiveness than those without suicidal ideation $\left(P<10^{-5}\right)$. (2) The three variables and the three dimensions of impulsiveness were significantly reciprocally correlated with each other $(P<0.01)$. (3) For the impulsiveness total score, non-planning, motor and attention, the multilinear regression models fit well (adjusted $R^{2}>0.3, S R M R<0.05$ ). Their indirect effects on suicidal ideation through depressive symptoms were all statistically significant (BIS-SDS-SIOSS, indirect effect coefficient=0.557, $P<0.001$; non-planning-SDS-SIOSS, indirect effect coefficient $=0.480, P<0.001$; motor-SDS-SIOSS, indirect effect coefficient $=0.486, P<0.001$; attention-SDS-SIOSS, indirect effect coefficient $=0.504, P<0.001)$. (4) Four parallel process latent growth curve models fit well (RMSEA<0.05). The total indirect effect of impulsiveness on the growth rate of suicidal ideation through the initial level and growth rate of depressive symptoms was significant (indirect effect coefficient $=-0.450, P=0.001$ ). The indirect effects of non-planning and attention impulsiveness were $-0.336(P=0.005)$ and $-0.314(P=0.002)$, respectively. However, the indirect effect of motor function on the growth rate of suicidal ideation was not significant (coefficient $=-0.379, P=0.067$ ).

Conclusions: This study mainly revealed that individuals with impulsive personalities might be at higher risk for and experience more rapid changes in suicidal ideation. The effects were indirect and mediated by depressive symptoms. This implies that impulsive personality with depressive symptoms is critical for suicide prevention and needs more attention.

\section{Background}

According to the newest worldwide statistics (1), nearly 800,000 people died from suicide in 2016, equating to 10.6 persons per 100,000. Additionally, there are still a large number of individuals who have attempted suicide or who have suicidal ideation and are at high risk for suicide completion. The definition of suicide is complicated and is divided into three phases: suicidal ideation, suicide attempts and lethal suicidal behaviour (2). For suicide prevention, it is critical to detect, manage, and intervene in both suicide attempts and ideation. There are many studies investigating risk factors for suicide attempts (3-9) but fewer investigations on suicidal ideation. Someone who attempts suicide has executed suicide preparation at least once. However, most individuals with suicidal ideation only exhibit thought in the brain. Detecting and managing suicidal ideation seem to decrease the risk much earlier than after a suicide attempt and may reduce the rate of attempts and even lethal suicidal behaviour.

Depression and impulsiveness are the two vital factors that convey high risk for suicide, including both attempt and ideation. Multiple studies have suggested that depression is associated with suicide. Among previous studies, major 
depressive disorder (MDD) and depressive symptoms were both included. One study revealed that among individuals with MDD, rates of suicidal ideation, suicide planning and suicide attempts were $43.6 \%, 13.7 \%$ and $12.3 \%$, respectively (10). Another study (8) discussed the differences in suicide attempts between groups with and without MDD. The results demonstrated that MDD individuals are more likely to write suicide notes and have high suicidal ideation levels. Their suicide motivation is to reduce mental pain, while individuals without MDD are more likely to exhibit impulsiveness and self-rescue characteristics. Their motivation is to threaten others. Other studies concentrate on the relationships between depressive symptoms and suicide. Depressive symptoms have been proven to be a promoting or risk factor for suicidal ideation, intent, and attempt (behaviour) (11-16). Regarding the risk or hazard ratio, epidemiological research of over one hundred thousand samples revealed a high hazard ratio of suicide death in terms of mild (2.18), moderate (2.13), severe (3.33) and extremely heavy (3.67) depressive symptoms (17). Another study reported a high odds ratio (3.4) for suicide among those with positive depressive symptoms compared to the negative group (18). Some researchers have tried to reveal the cognitive neural basis of the relationship between depression and suicide. Research on suicide attempters with MDD has shown that compared to individuals who have not attempted suicide, those who have attempted suicide present larger squares and cortical volumes in the postcentral area of the left cerebral hemisphere and lateral region of the occipital lobe and smaller squares in the superior frontal area of the left cerebral hemisphere (19).

Studies on impulsiveness and suicide have revealed a close association between these phenomena, although the definitions of impulsiveness differ. Early research has shown that one-quarter of suicidal behaviours are impulsive (20). There are at least three kinds of impulsiveness among these studies: impulsive personality (and related disorders), impulse control disorder and impulsive behaviour. Impulsive personality is associated with suicide in several dimensions. One study applied the Big Five personality structure and determined that among young groups, agreeableness was negatively correlated with suicidal ideation, while neuroticism and openness to experience were positively correlated with suicidal ideation. Neuroticism is associated with suicide across age groups (21). Neuroticism reflects emotional impulsiveness. Some studies have shown that impulsive personality is a risk factor for both suicidal ideation and attempts $(22,23)$. Others have combined impulsiveness with additional factors and reached similar conclusions $(24,25)$. Among these factors, aggression is a cofactor with impulsivity for suicide, which has been supported by several studies $(5,24-26)$. Impulsiveness is associated with suicide in terms of both traits (27) and personality disorder (28), while both trait impulsiveness and personality disorders are not consistently considered risk factors for suicide (28). Impulse control disorder is correlated with suicide, as revealed by both epidemiological data (29) and neural biochemical evidence (30). Impulsive behaviour is usually examined in studies by decision making (gambling) or cognitive control behavioural tasks. Although there are not only impulsive components in these tasks, the task completion quality is related to impulsiveness. Studies have shown that both gambling tasks and cognitive task completion are correlated with suicide attempts $(31,32)$. There are also other structures representing impulsiveness associated with suicide, such as attention deficit (e.g., attention deficithyperactivity disorder, ADHD) (26) and substance abuse (33). Impulsive-related disorders (including impulse control disorder and other disorders with impulsive structure) and impulsive behaviour are both based on an impulsive personality, which can include a wide spectrum of impulsive dimensions. Barratt constructed a theoretical structure that divides impulsiveness into three dimensions, namely, non-planning impulsiveness, motor impulsiveness and attention impulsiveness, and thus developed the Barratt (34) Impulsiveness Scale (BIS). With updates to the scale version, opinions about its factor structure change. After the 10th version, each of the three dimensions was divided into two factors. Non-planning consists of self-control and cognitive complexity. Motor dimension includes motor and perseverance. The attention dimension includes attention and cognitive instability. Therefore, the latest version of the BIS mainly includes most of the research aspects of impulsiveness. 
There is much evidence that depression and impulsiveness are independently related to suicide. Some evidence indicates that these two factors can work together to influence suicide. Compared to individuals with affect disorders only, those who both have affect disorder and substance use disorder have a higher risk of Type $B$ personality disorder and are more aggressive and impulsive, with increased suicide attempts and suicide lethality (9). This implies that depression and impulsiveness interactively affect suicide at the level of disorder. Another study demonstrated that MDD is more frequently detected in suicide planning rather than impulsive suicide (35), indicating that depression and impulsiveness result in different types of suicide behaviours. Regarding the level of behaviour mode, research on decision making has been conducted, revealing that among depressive individuals, worse decision-making ability (related to impulsiveness) predicts suicide attempts (3). MacGregor (6) conducted a longitudinal study, and the results showed that avoidant attachment was correlated with both impulsiveness and MDD, while impulsiveness was correlated with suicide attempts. This indicates that impulsiveness and depression may be correlated with each other, influencing suicide together. Nevertheless, these two factors do not interactively affect suicide. Another study indicated that impulsiveness is related to MDD but not to other clinical features, including suicide (36).

Existing research has demonstrated that depression and impulsiveness can predict suicide attempts or ideation independently, while how these two factors influence suicide together is not clear, and there is currently not a consistent conclusion on this matter. The reason for this might be due to the existence of various types of impulsiveness and unclear definitions of depression. Moreover, suicide as the outcome variable is not definitively linked to either behaviour or attempt. As suicidal ideation logically occurs earlier than attempts, which is meaningful for early suicide prevention, the present study focuses on suicidal ideation. Additionally, as Barratt's impulsiveness structure includes most of the concerned dimensions of impulsiveness, we test participants' impulsiveness with the Barratt Impulsiveness Scale (BIS). The aims of the present study are to define whether Barratt's structured impulsiveness and depressive symptoms are correlated with suicidal ideation and to determine predictive paths from the two factors to suicidal ideation. These results may shed light on the early prevention and detection of probable suicidal behaviours. Given that the cross-sectional prediction model does not provide sufficient reliable evidence to establish a causal relationship between variables, the present study combines cross-sectional and longitudinal methods to test these relationships. According to the research objectives, we first conduct a variance comparison to test the hypothesis that those who had suicidal ideation exhibited increased severe depressive symptoms and a higher level of impulsiveness than those who did not have suicidal ideation. Second, we hypothesize that impulsiveness positively predicts suicidal ideation mediated by depressive symptoms in the crosssectional analysis. Third, we apply a longitudinal growth model to test the hypothesis that impulsiveness predicts the growth rate of suicidal ideation in an inter-temporal development process, which is mediated by the initial level and growth rate of depressive symptoms.

\section{Methods}

\section{Participants}

All data were derived from the annual health examination (psychological test) of civilian undergraduate students at one medical university in Chongqing, China. A cluster sampling method was used to select a cohort of participants' psychological test data.

Samples for cross-sectional analysis were collected from testing data in the first semester of the 2019-2020 academic year. All data were initially collected from November to December 2019. For longitudinal analysis, 
samples were collected from testing data in three periods: the entrance test (at the beginning of the first semester of the 2019-2020 academic year), the end-of-semester test (at the end of the first semester of the 2019-2020 academic year) and the beginning-of-semester test (at the beginning of the second semester of the 2019-2020 academic year). Each period was approximately three months apart, and the periods were defined as three waves: Wave 0 , Wave 1 , and Wave 2.

In the cross-sectional analysis, 480 participants were examined, including two grades of students (freshman and sophomore). There were no reported mental illnesses or related family histories. In the longitudinal analysis, 198 participants were initially included from the freshmen of 2019. Because of lack of follow-up, two cases were missing in Wave 1, and 19 cases were missing in Wave 2. Therefore, 177 cases remained for further analysis.

\section{Measures}

\section{Suicidal ideation}

Suicidal ideation was assessed by the Self-rating Idea of Suicide Scale (SIOSS). This scale was developed in Chinese by Xia (37). Twenty-six items were included, and subjects chose yes or no concerning whether they had symptoms or ideas described in the items. The scale was divided into four subscales: desperation, optimism, sleep, and lie. Suicidal ideation was represented by the summed scores of desperation, optimism, and sleep. Higher scores implied stronger suicidal ideation. The SIOSS has good reliability and validity in the Chinese population (38). According to the Chinese norm, those whose summed scores were $\geq 12$ were regarded as having suicidal ideation (39).

\section{Impulsiveness}

The Barratt Impulsiveness Scale was originally developed by Ernest S. Barratt in 1959 with the aim of evaluating one's behavioural patterns or personality traits of impulsiveness (34). From the $10^{\text {th }}$ version on, Barratt divided the construct of impulsiveness into three dimensions: non-planning impulsiveness, motor impulsiveness, and attention impulsiveness, representing lack of forethought, acting without thinking, and making quick decisions, respectively (34). The $11^{\text {th }}$ Chinese version of the BIS was revised under the context of Chinese culture and retained good reliability and validity $(40)$.

\section{Depressive symptoms}

The Self-rating Depression Scale (SDS) was initially developed by William W. K. Zung in 1965 for evaluating depressive symptoms in patients with depression disorder (41). The scale consisted of 20 items with 1- to 4-point Likert scale values, including diagnostic criteria for the presence of a pervasive depressed affect and physiological and psychological concomitants. It can also be used for preliminary screening in outpatient clinics (42), early detection of depression (43) or depressive state evaluation in the general population $(44,45)$. The Chinese cooperative group developed the locative version of the SDS and interviewed 1,340 healthy cases defining the first Chinese norm, of which the average raw score was 33.46 \pm 8.55 (46). The Chinese version of the SDS has demonstrated good reliability and validity across various groups of people.

\section{Statistical analyses}

Variance tests were conducted in SPSS 20.0 (47) using a non-parametric test between two independent variables. Cross-sectional mediation effect tests were conducted in SmartPLS 3.0 (48). Impulsiveness (including all three of its 
dimensions) was set as the predicting variable. Depressive symptoms were set as the mediator, while suicidal ideation was regarded as the outcome. Longitudinal analysis was conducted in Mplus 8.3 (49) with a parallel process latent growth curve model (LGCM) and a mediating effect test. The parallel process LGCM was used to analyse the reciprocal intertemporal effect between the intercept and slope of two changing variables. During analysis, several covariates might be included. Previous studies $(50,51)$ regarded one of the parallel variables as a mediator and the covariate as a predictor; thus, the mediating effect was included in this model. In the present longitudinal analysis, impulsiveness and its three dimensions at baseline were set as predictors. Depressive symptoms (both the initial level and the growth rate) were regarded as one of the parallel growing variables and the mediator. The growth rate of suicidal ideation was set as another parallel growing variable and outcome. Mediating effects in these two models were both examined with the bootstrap method with a sampling size of 5,000.

\section{Results}

\section{Demographic characteristics}

In the cross-sectional analysis, the cohort included 245 females (51.0\%) and 235 males (49.0\%) aged $16 \sim 21$ (18.66 \pm 0.80$)$. In the longitudinal analysis, the cohort included 107 females (60.5\%) and 70 males (39.5\%) aged 16 $\sim 20$ (17.80 \pm 0.68$)$ at baseline (the beginning of the 2019-2020 academic semester). All demographic characteristics were collected during the first psychological test, including grade, residence, only child status, and family structure, as shown in Table 1. 
Table 1

Demographic characteristics of cross-sectional and longitudinal analysis

\begin{tabular}{|c|c|c|c|c|}
\hline & \multicolumn{2}{|c|}{$\begin{array}{l}\text { Cross-sectional analysis } \\
(n=480)\end{array}$} & \multicolumn{2}{|c|}{$\begin{array}{l}\text { Longitudinal analysis } \\
(n=177)\end{array}$} \\
\hline & $\mathrm{N}(\%)$ & Mean (SD) & $\mathrm{N}(\%)$ & Mean (SD) \\
\hline Age & $480(100)$ & $18.66(0.80)$ & $177(100)$ & $17.80(0.68)$ \\
\hline Gender (male) & $235(49.0)$ & & $70(39.5)$ & \\
\hline Gender (female) & $245(51.0)$ & & $107(60.5)$ & \\
\hline Grade (freshman) & $196(40.8)$ & & $177(100)$ & \\
\hline Grade (sophomore) & $284(59.2)$ & & $\mathrm{N} / \mathrm{A}$ & \\
\hline Residence (urban) & $387(80.6)$ & & $139(78.5)$ & \\
\hline Residence (rural) & $93(19.4)$ & & $38(21.5)$ & \\
\hline Only child & $273(56.9)$ & & $100(56.5)$ & \\
\hline Non-only child & $207(43.1)$ & & $77(43.5)$ & \\
\hline Family structure (joint family) & $146(30.4)$ & & $47(26.6)$ & \\
\hline Family structure (nuclear family) & $298(62.1)$ & & $116(65.5)$ & \\
\hline Family structure (broken family) & $36(7.5)$ & & $14(7.9)$ & \\
\hline Left-behind child (yes) & $73(15.2)$ & & $28(15.8)$ & \\
\hline Left-behind child (no) & $377(78.5)$ & & $149(84.2)$ & \\
\hline Left-behind child (null) & $30(6.3)$ & & $\mathrm{N} / \mathrm{A}$ & \\
\hline
\end{tabular}

Comparison of impulsiveness and depressive symptoms between participants with and without suicidal ideation

In accordance with the SIOSS scores, participants were divided into 2 groups, those with suicidal ideation $(n=28)$ vs. those without suicidal ideation $(n=452)$. Given that neither the BIS (including three dimensions: non-planning, motor and attention) nor the SDS scores fit a normal distribution in the group without suicidal ideation (K-S $=0.044$, $0.052,0.056,0.076,0.056, P=0.038,0.006,0.002,0.000,0.002)$, we performed a non-parametric test to investigate the variance in BIS and SDS scores between the two groups. The results showed that compared to participants without suicidal ideation, participants with suicidal ideation presented higher BIS and SDS scores. Specifically, the three subscales of BIS (non-planning, motor and attention) had similar results, as shown in Table 2. 
Table 2

BIS and SDS score comparison between the two groups showing the median (IQR)

\begin{tabular}{|lllll|}
\hline & $\begin{array}{l}\text { With suicidal ideation } \\
(\mathbf{n = 2 8})\end{array}$ & $\begin{array}{l}\text { Without suicidal ideation } \\
(\mathbf{n = 4 5 2})\end{array}$ & $\boldsymbol{P} / \mathrm{Z}$ \\
\hline SDS & $64.00(12.00)$ & $45.00(15.00)$ & $685.00 /-7.93$ & $<10^{-14}$ \\
\hline BIS & $51.67(20.00)$ & $34.17(15.00)$ & $2126.50 /-5.90$ & $<10^{-8}$ \\
\hline non-planning & $55.00(21.90)$ & $35.00(22.50)$ & $2736.50 /-5.05$ & $<10^{-6}$ \\
\hline motor & $51.25(25.60)$ & $32.50(17.50)$ & $2212.00 /-5.79$ & $<10^{-8}$ \\
\hline attention & $50.00(22.50)$ & $35.00(15.00)$ & $3138.50 /-4.49$ & $<10^{-5}$ \\
\hline Note: IQR, inter-quartile range; U/Z, Mann-Whitney U test statistic/Z-score & \\
\hline
\end{tabular}

Mediating effect of depressive symptoms on the relationship between impulsiveness and suicidal ideation

Prior to the mediating effect analysis, relationships between the SIOSS, SDS, BIS, and its three subscale scores were analysed. Using Spearman correlation coefficients due to the non-normal distribution of variables, we found that all variables were significantly correlated with each other, as shown in the following correlation matrix (Table 3).

According to the coefficients, the SIOSS scores were more highly correlated with the SDS than with the BIS and its subscales. The correlation between the SDS and BIS was relatively stronger than the correlation between the SIOSS and BIS (similar for the three BIS subscales). This indicated that our hypothesis that depressive symptoms were mediators could examined.

Table 3

Correlation matrix between the SIOSS, SDS, BIS, and its three subscales

\begin{tabular}{|c|c|c|c|c|c|c|}
\hline & 1 & 2 & 3 & 4 & 5 & 6 \\
\hline 1 & 1.000 & & & & & \\
\hline 2 & 0.476 ** & 1.000 & & & & \\
\hline 3 & $0.385^{\star \star}$ & $0.888 * *$ & 1.000 & & & \\
\hline 4 & $0.440 * \star$ & $0.727 * \star$ & $0.427 * \star$ & 1.000 & & \\
\hline 5 & $0.323^{\star \star}$ & $0.803^{\star *}$ & 0.710 ** & 0.381 ** & 1.000 & \\
\hline 6 & 0.726 ** & $0.633^{\star \star}$ & $0.525^{\star \star}$ & $0.525^{\star \star}$ & $0.506^{\star *}$ & 1.000 \\
\hline
\end{tabular}

Applying a mediating effect model, the SIOSS score was set as the dependent variable, and the BIS score and its subscale scores were set as independent variables. The SDS score was set as the mediator. Therefore, we developed four regression models: (1) BIS-SDS-SIOSS; (2) non-planning-SDS-SIOSS; (3) motor-SDS-SIOSS; and (4) attention-SDS-SIOSS. All were latent variables, while the observed variables were the item scores of each scale. The 
fit indexes of the 4 models are shown in Table 4. The data revealed that all models seemed to be well identified, implying a strong mediating effect of the SDS score.

Table 4

Fit indexes of four linear regression models with mediators

\begin{tabular}{|c|c|c|c|c|c|c|c|c|}
\hline \multirow[t]{2}{*}{ indexes } & \multicolumn{2}{|l|}{ Model 1} & \multicolumn{2}{|l|}{ Model 2} & \multicolumn{2}{|l|}{ Model 3} & \multicolumn{2}{|l|}{ Model 4} \\
\hline & depression & $\begin{array}{l}\text { suicide } \\
\text { ideation }\end{array}$ & depression & $\begin{array}{l}\text { suicide } \\
\text { ideation }\end{array}$ & depression & $\begin{array}{l}\text { suicide } \\
\text { ideation }\end{array}$ & depression & $\begin{array}{l}\text { suicide } \\
\text { ideation }\end{array}$ \\
\hline $\begin{array}{l}\text { Adjusted } \\
\mathrm{R}^{2}\end{array}$ & 0.522 & 0.602 & 0.367 & 0.600 & 0.403 & 0.612 & 0.417 & 0.600 \\
\hline$P$ & 0.000 & 0.000 & 0.000 & 0.000 & 0.000 & 0.000 & 0.000 & 0.000 \\
\hline SRMR & 0.040 & & 0.042 & & 0.042 & & 0.042 & \\
\hline
\end{tabular}

The total explained variances of the four models were all greater than 60\% (SRMR, standard root mean square residual, where a value below 0.05 indicates a well-identified model).

From the path diagrams, a complete mediating effect was shown to exist for the SDS score between the BIS and SIOSS scores. In addition, the SDS score had a mediating effect between the three BIS subscale scores and the SIOSS score (see Fig. 1).

\section{Mediating effect of depressive symptoms on the relationship between impulsiveness and the growth of suicidal ideation in the parallel process LGCM}

As a cross-sectional study can only reveal a static effect at a given time and does not sufficiently explain the causal relationship between the variables, we conducted a longitudinal analysis to investigate the mediating effect in an inter-temporal causal model to explain the effect of impulsiveness on changes in suicidal ideation through depressive symptoms. First, we constructed a simple latent growth curve model of suicidal ideation with covariate impulsiveness (tested in Wave 1) and set it as the baseline model (Model 1). However, the model fit was not good because the index, the root mean square error of approximation (RMSEA), was greater than 0.1 (52). Next, we included depressive symptoms as an indirect variable that concurrently grows with suicidal ideation, which were both predicted by impulsiveness. As a result, the model fit was optimized but still did not reach the experiential criterion (RMSEA < 0.08). Next, several demographic factors (gender, only child, residence, family structure and leftbehind child) were included in the model to be controlled as covariates for suicidal ideation, and a well-identified model was created. The optimized model fit indexes are shown in Table 5. Following Model 3, three models were established for the three dimensions' effects on depressive symptoms and suicidal ideation. For all of models, the SDS and BIS scores were centralized. The model fit indexes are presented in Table 6.

Table 5

Model fit optimization

\begin{tabular}{|lllll|}
\hline Model & $\chi 2 / \mathrm{df}, \boldsymbol{P}$ & RMSEA [95\%Cl] & CFI/TLI & SRMR \\
\hline Baseline (Model 1) & $12.605 / 2,0.0018$ & $0.173[0.0900 .270]$ & $0.941 / 0.823$ & 0.068 \\
\hline Model 2 & $21.919 / 7,0.0026$ & $0.110[0.0600 .163]$ & $0.978 / 0.934$ & 0.039 \\
\hline Model 3 & $48.803 / 37,0.0927$ & $0.042[0.0000 .072]$ & $0.983 / 0.976$ & 0.063 \\
\hline
\end{tabular}


Table 6

Model fit indexes of three-dimensional effects on suicidal ideation mediated through depressive symptoms

\begin{tabular}{|lllll|}
\hline Model & X2/df, $P$ & RMSEA [95\%Cl] & CFI/TLI & SRMR \\
\hline$(1)$ & $45.756 / 37,0.1531$ & $0.037[0.0000 .068]$ & $0.987 / 0.981$ & 0.064 \\
\hline$(2)$ & $47.195 / 37,0.1216$ & $0.039[0.0000 .070]$ & $0.984 / 0.979$ & 0.065 \\
\hline$(3)$ & $49.701 / 37,0.0792$ & $0.044[0.000$ 0.073] & $0.980 / 0.973$ & 0.067 \\
\hline \multicolumn{2}{|l|}{ Note: (1) Non-planning-(isds, ssds)-ssioss; (2) Motor-(isds, ssds)-ssioss; (3) Attention-(isds, ssds)-ssioss; } \\
\hline \multicolumn{2}{|l|}{ isioss: intercept of the SIOSS growth curve; ssioss: slope of the SIOSS growth curve } \\
\hline \multicolumn{2}{|l}{ isds: intercept of SDS growth curve; ssds: slope of SDS growth curve } \\
\hline
\end{tabular}

Specifically, the path of each correlation shown in Fig. 2 revealed that impulsiveness affects the growth of suicidal ideation in total through both the initial level and the growth of depressive symptom (according to Fig. 2(A)); namely, the indirect effects were significant. On the other hand, impulsiveness directly affected the growth of suicidal ideation, but the direct and indirect effects had opposite signs. Similar results were observed for the effects of non-planning and attention impulsiveness (Fig. 2(B) and 2(D)). For motor impulsiveness, the direct and indirect effects were not significant.

\section{Discussion}

The present study investigated the predicted effects of depressive symptoms and impulsiveness on suicidal ideation. First, the results revealed that suicidal ideation significantly differentiated depressive symptoms and impulsiveness scores. Specifically, individuals with suicidal ideation scored higher than those who did not have suicidal ideation both on depressive symptoms and on impulsiveness. This agrees with the results of previous studies, although many of them focused on suicide attempts, depressive disorders, and impulsive disorders.

In terms of depression, individuals with disorders primarily commit suicide to reduce their pain, while those who are not psychopathic commit suicide for impulsiveness and to threaten others (8). Therefore, similar correlations of depressive disorder and depressive symptoms with suicide may have different mechanisms. Some previous studies verify this point of view. Kim's study on impulsive suicide and non-impulsive suicide demonstrated that MDD is less frequent in impulsive suicide (35). In Wei's results, individuals characterized by impulsive suicide have a lower prevalence of psychopathy (33). Another study concluded that suicide attempters without psychopathy exhibit more impulsivity than those with other disorders (53). Depressive symptoms that are subclinical are related to impulsiveness more closely than to depressive disorder. Therefore, the present study sampled a generally healthy group. These results demonstrate that depressive symptoms are positively correlated with impulsiveness and that both are correlated with suicide. Furthermore, based on these results, depression and impulsiveness are both predictors for suicide, while depressive symptoms were additionally influenced by impulsiveness.

Previous studies have examined many aspects of the connotation and extension of impulsiveness. An impulsive personality with aggression $(5,26)$ is thought to be the reason for suicidal behaviours, while attention deficit (ADHD) (26) and impulse control disorders $(29,30)$ are also risk factors for suicide. In the present study, impulsiveness consisted of three dimensions: non-planning, motor and attention. All three dimensions indirectly influenced suicidal ideation through depressive symptoms. Motor impulsiveness refers to those who do not control their behaviours 
(34). This agrees with studies on impulse control disorders, although motor impulsiveness is a component of personality. Attention impulsiveness means that one cannot continue to pay attention to specific stimuli or events (34), which is similar to results from studies on ADHD. Although motor and attention impulsiveness are still far from psychopathy or other disorders, impulsive personality is related to impulsive-related disorders and thus is associated with suicidal behaviour. Prior to mental and behavioural impairment, an impulsive personality is already associated with suicidal ideation. Moreover, non-planning is described as a trait that one cannot plan rationally before behaviours (34). This means that one acts without deep thought. Therefore, non-planning is more likely classified as a trait of thinking. The present study's result that non-planning indirectly affects suicidal ideation without a direct effect indicates that it results in depressive symptoms first, followed by suicidal ideation. This conclusion coincides with the research result that impulsiveness makes people more likely to be exposed to stress or negative events and thus results in suicide (25).

Regarding suicide attempts, it is relatively easy to discriminate positive and negative external behaviours. The operational definition of the severity of a suicide attempt varies in different studies, including the times of suicide behaviours (9), medical seriousness (5), prevalence (7) and lethality (28). It is difficult to include these aspects into a single component. There is research indicating that impulsiveness does not predict the severity of suicide attempts (5). The present study considered suicidal ideation an outcome, which was a latent variable and needed to be tested with a scale. As a continuous variable, suicidal ideation severity is measured by scale scores. These results demonstrate that depressive symptoms and impulsiveness were both positively correlated with suicidal ideation. This means that these two factors positively predict the severity of suicidal ideation.

In the present study, we analysed three variables together with a correlation matrix and regression method, and all of the variables were included. In many previous studies, one of the variables was regarded as a group (36, 54-57). A predictive model built to include impulsive personality, depressive symptoms and suicidal ideation shows that impulsive personality and depressive symptoms can explain the variance of suicidal ideation independently, but this model does not mention the correlation between impulsiveness and depressive symptoms (58). Based on our result that suicidal ideation was more highly correlated with depressive symptoms than was impulsiveness (including the three dimensions), while the correlations between depressive symptoms and impulsiveness (including the three dimensions) were relatively higher than those between suicidal ideation and impulsiveness (including the three dimensions), we deduced that impulsiveness has a stronger indirect effect on suicidal ideation than direct impulsiveness. The results of regression models indicated that for total impulsiveness, including non-planning, motor and attention impulsiveness, depressive symptoms were mediators of suicidal ideation. These results are in agreement with the "stress vulnerability model" theory posited by Bonner and Rich in 1987 (59). According to this model, suicide is affected by both vulnerability (personality, emotion, cognition, etc.) (60) and stress (life events, environment, etc.) at the same time. These two factors existing together increases the risk of suicidal ideation. In previous studies, personality, especially neuroticism, was definitely vulnerable (61), while depression was regarded as emotional vulnerability (62). However, in the present study, depressive symptoms were set as a stressor because they were more a state than a trait. The mediation model demonstrated that impulsiveness was vulnerable not only to suicide but also to depressive symptoms. All impulsiveness dimensions influenced suicidal ideation indirectly. However, the results could still not define whether the inner mechanisms of the three dimensions' effects were the same.

To confirm the causal relationships between impulsiveness and the growth of depressive symptoms as well as suicidal ideation, a longitudinal analysis was conducted followed by a cross-sectional mediation effect analysis. The results showed that impulsiveness had an indirect effect on the growth rate of suicidal ideation through the

Page $11 / 20$ 
initial level and growth rate of depressive symptoms. The paths in the LGCM (Fig. 2(A)) indicated that impulsiveness directly and positively affected the growth of suicidal ideation. At the same time, indirect and negative predicting paths existed from impulsiveness to the growth of suicidal ideation. Specifically, increased impulsiveness predicted a higher initial level of depressive symptoms and a more rapid decline in suicidal ideation. At the same time, impulsiveness predicted a faster decrease in suicidal ideation through a slower increase in depressive symptoms. As a result, impulsiveness indirectly negatively influenced the growth of suicidal ideation. Because the indirect negative effect was larger than the direct positive effect, the total effect of impulsiveness on suicidal ideation was negative. Similar effects were observed for non-planning and attention impulsiveness. The total effect of motor function on suicidal ideation was not significant. This might indicate that the inner mechanisms of the three kinds of impulsiveness' effects on suicidal ideation differ. In the present study, the severity of suicidal ideation decreased as a whole across the three waves, declining more quickly for individuals with stronger impulsiveness. This indicates that personality impulsiveness may result in inconsistent risk for suicidal ideation variance over a short time. It is difficult to detect a quickly changing state. Therefore, the impulsiveness personality criterion provides a relatively stable observed indicator. However, this study does not provide increasing suicidal ideation data, which may be more meaningful and evidential as a reference for prevention.

Despite the supported hypothesis, this study still has some limitations. First, there were only three waves in the longitudinal study, which met the lowest criteria and might have conveyed inconsistency in the results. Second, although some of the paths were significant, the effect sizes were relatively low (below 0.3 ). Third, the participants included in the samples were limited to college students, so the effect is uncertain in other demographics. Therefore, future studies need to include more waves in the longitudinal analyses to increase the statistical consistency. More samples from a variety of groups, such as middle school students, adults, and elderly individuals, should also be included. Moreover, other methods, such as electroencephalogram (EEG) and functional magnetic resonance imaging (fMRI), can be administered to verify the indirect effects more precisely and to identify the mechanisms among them.

\section{Conclusions}

This study revealed that individuals with or without suicidal ideation exhibit differences in depressive symptoms and impulsiveness. In addition, the study demonstrated that depressive symptoms and impulsiveness are both related to suicidal ideation. Impulsiveness indirectly and positively affects suicidal ideation through depressive symptoms and negatively influences the total growth of suicidal ideation through the initial level and growth rate of depressive symptoms. The causal and mediating relationship between these two factors and suicidal ideation was identified. In practice, these results illustrate that individuals with impulsive personalities may be at higher risk and exhibit faster changes in suicidal ideation. This implies that in suicide prevention, impulsive personality with depressive symptoms is critical and needs more attention.

\section{List Of Abbreviations}

SDS: Self-rating Depression Scale

BIS: Barratt Impulsiveness Scale

SIOSS: Self-rating Idea of Suicide Scale

SRMR: standardized root mean square residual 
RMSEA: root mean square error of approximation

MDD: major depressive disorder

ADHD: attention deficit-hyperactivity disorder

LGCM: latent growth curve model

IQR: inter-quartile range

U/Z: Mann-Whitney U test statistic/Z-score

CFI/TLI: comparative fit index/ Tucker-Lewis index

isds: intercept of growth curve of SDS

isioss: intercept of growth curve of SIOSS

ssds: slope of growth curve of SDS

ssioss: slope of growth curve of SIOSS

EEG: electroencephalogram

fMRI: functional magnetic resonance imaging

\section{Declarations}

\section{Availability of data and materials}

All data used and/or analyzed in this study are available from the corresponding author upon request.

\section{Authors' contributions}

The author Jingxuan Zhang contributed to the design of the research, conducting statistical analyses and drafting the manuscript. Xiaolin Zhang contributed to the arranging of materials and data, conducting statistical analyses and editing manuscript. Guoyu Yang contributed to the longitudinal study design, data processing and manuscript revising. Zhengzhi Feng contributed to the design of the whole study and critical revising of the manuscript. All authors have read and revised the final manuscript.

\section{Ethics approval and consent to participate}

The Medical Ethics Committee of Army Medical University permitted this study. All methods were performed in accordance with the Declaration of Helsinki and other relevant guidelines and regulations. All participants provided informed consent.

\section{Consent for publication}

Not applicable. 
The authors declare that they have no competing interests.

\section{Acknowledgements}

We thank all the co-authors and participants who contributed to this research. We also thank the Psychological Training Centre of Army Medical University for data management.

\section{Funding}

This research was supported by the National Natural Science Foundation of China (81971278).

\section{References}

1. World-Health-Organization. World health statistics 2020: monitoring health for the SDGs, sustainable development goals. 2020. https://www.who.int/iris/bitstream/handle/10665/332070/9789240005105eng.pdf. Licence: CC BY-NC-SA 3.0 IGO. Accessed 20 Apr. 2020.

2. Du R, Jiang G. Suicidal behaviors: Risk factor, psychological theory and future research. Advances in Psychological Science. 2015;23(8):1437-52.

3. Clark L, Dombrovski AY, Siegle GJ, Butters MA, Shollenberger CL, Sahakian BJ, et al. Impairment in risk-sensitive decision-making in older suicide attempters with depression. Psychol Aging. 2011;26(2):321-30.

4. Salvatore P, Baldessarini RJ, Khalsa HM, Indic P, Maggini C, Tohen M. Negative affective features in 516 cases of first psychotic disorder episodes: Relationship to suicidal risk. J Depress Anxiety. 2013;2(1):1000131.

5. Gvion Y, Horresh N, Levi-Belz Y, Fischel T, Treves I, Weiser M, et al. Aggression-impulsivity, mental pain, and communication difficulties in medically serious and medically non-serious suicide attempters. Compr Psychiatry. 2014;55(1):40-50.

6. MacGregor EK, Grunebaum MF, Galfalvy HC, Melhem N, Burke AK, Brent DA, et al. Depressed parents' attachment: effects on offspring suicidal behavior in a longitudinal family study. J Clin Psychiatry. 2014;75(8):879-85.

7. Izci F, Findikli EK, Zincir S, Zincir SB, Koc MI. The differences in temperament-character traits, suicide attempts, impulsivity, and functionality levels of patients with bipolar disorder I and II. Neuropsychiatr Dis Treat. 2016;12:177-84.

8. Wei S, Li H, Hou J, Chen W, Chen X, Qin X. Comparison of the characteristics of suicide attempters with major depressive disorder and those with no psychiatric diagnosis in emergency departments of general hospitals in China. Ann Gen Psychiatry. 2017;16:44.

9. Rizk MM, Galfalvy H, Miller JM, Milak M, Parsey R, Grunebaum M, et al. Characteristics of depressed suicide attempters with remitted substance use disorders. J Psychiatr Res. 2020. doi: 10.1016/j.jpsychires.2020.10.041

10. Subramaniam M, Abdin E, Els S, Louisa P, Chong SA. Suicidal Ideation, Suicidal Plan and Suicidal Attempts Among Those with Major Depressive Disorder. Annals of the Academy of Medicine, Singapore. 2014;43(8):412-21.

11. Testa CR, Steinberg L. Depressive symptoms and health-related risk-taking in adolescence. Suicide Life Threat Behav. 2010;40(3):298-305.

12. Cukrowicz KC, Schlegel EF, Smith PN, Jacobs MP, Van Orden KA, Paukert AL, et al. Suicide ideation among college students evidencing subclinical depression. J Am Coll Health. 2011;59(7):575-81. 
13. Campos RC, Santos S, Piteira M, Abreu M, Tavares S. Interpersonal Needs, Depressive Symptoms, and Suicide Ideation in a Sample of Portuguese Elderly Patients Recovering from Acute Medical Conditions. J Clin Psychol Med Settings. 2018;25(1):1-10.

14. Nascimento VSD, Santos AVD, Arruda SB, Silva GAD, Cintra JDS, Pinto TCC, et al. Association between eating disorders, suicide and depressive symptoms in undergraduate students of health-related courses. Einstein (Sao Paulo). 2020;18:eA04908.

15. Pera A. Depressive Symptoms, Anxiety Disorder, and Suicide Risk During the COVID-19 Pandemic. Front Psychol. 2020;11:572699.

16. Ayar D, Sabanciogullari S. The effect of a solution-oriented approach in depressive patients on social functioning levels and suicide probability. Perspect Psychiatr Care. 2021;57(1):235-45.

17. Yi SW. Depressive Symptoms on the Geriatric Depression Scale and Suicide Deaths in Older Middle-aged Men: A Prospective Cohort Study. J Prev Med Public Health. 2016;49(3):176-82.

18. Veras JL, Ximenes RC, de Vasconcelos FM, Sougey EB. Prevalence of Suicide Risk Among Adolescents With Depressive Symptoms. Arch Psychiatr Nurs. 2016;30(1):2-6.

19. Kang SG, Cho SE, Na KS, Lee JS, Joo SW, Cho SJ, et al. Differences in brain surface area and cortical volume between suicide attempters and non-attempters with major depressive disorder. Psychiatry Res Neuroimaging. 2020;297:111032.

20. Wyder M, De Leo D. Behind impulsive suicide attempts: indications from a community study. J Affect Disord. 2007;104:167-73.

21. Na KS, Cho SE, Hong JP, Lee JY, Chang SM, Jeon HJ, et al. Association between personality traits and suicidality by age groups in a nationally representative Korean sample. Medicine (Baltimore). 2020;99(16):e19161.

22. Zhang Y. The Study in the Relationship between Impulsive Personality Traits and Suicide. Advances in Psychology. 2014;04(4):537-44.

23. Tan L, Xia T, Reece C. Social and individual risk factors for suicide ideation among Chinese children and adolescents: A multilevel analysis. Int J Psychol. 2018;53(2):117-25.

24. McGirr A, Renaud J, Bureau A, Seguin M, Lesage A, Turecki G. Impulsive-aggressive behaviours and completed suicide across the life cycle: a predisposition for younger age of suicide. Psychol Med. 2008;38(3):407-17.

25. Jordan JT, Samuelson KW, Tiet QQ. Impulsivity, Painful and Provocative Events, and Suicide Intent: Testing the Interpersonal Theory of Suicide. Suicide Life Threat Behav. 2019;49(4):1187-95.

26. Conejero I, Jaussent I, Lopez R, Guillaume S, Olie E, Hebbache C, et al. Association of symptoms of attention deficit-hyperactivity disorder and impulsive-aggression with severity of suicidal behavior in adult attempters. Sci Rep. 2019;9:4593.

27. May AM, Klonsky ED. "Impulsive" suicide attempts: What do we really mean? Personal Disord. 2016;7(3):293302.

28. Rimkeviciene J, O'Gorman J, De Leo D. Impulsive suicide attempts: a systematic literature review of definitions, characteristics and risk factors. J Affect Disord. 2015;171:93-104.

29. da Silveira DX, Fidalgo TM, Di Pietro M, Santos JG, Jr., Oliveira LQ. Is drug use related to the choice of potentially more harmful methods in suicide attempts? Subst Abuse. 2014;8:41-3.

30. Lidberg L, Belfrage H, Bertilsson L, Evenden MM, Asberg M. Suicide attempts and impulse control disorder are related to low cerebrospinal fluid 5-HIAA in mentally disordered violent offenders. Acta Psychiatr Scand. 
2000;101(5):395-402.

31. Ismael C, Emilie O, Philippe C, Raffaella C. Suicide in older adults: current perspectives. Clinical Interventions in Aging. 2018;13:691-9.

32. Wyart M, Jaussent I, Ritchie K, Abbar M, Jollant F, Courtet P. lowa Gambling Task Performance in Elderly Persons with a Lifetime History of Suicidal Acts. Am J Geriatr Psychiatry. 2016;24(5):399-406.

33. Wei S, Liu L, Bi B, Li H, Hou J, Chen W, et al. Comparison of impulsive and nonimpulsive suicide attempt patients treated in the emergency departments of four general hospitals in Shenyang, China. Gen Hosp Psychiatry. 2013;35(2):186-91.

34. Stanford MS, Mathias CW, Dougherty DM, Lake SL, Anderson NE, Patton JH. Fifty years of the Barratt Impulsiveness Scale: An update and review. Personality \& Individual Differences. 2009;47(5):385-95.

35. Kim J, Lee KS, Kim DJ, Hong SC, Choi KH, Oh Y, et al. Characteristic Risk Factors Associated with Planned versus Impulsive Suicide Attempters. Clin Psychopharmacol Neurosci. 2015;13(3):308-15.

36. Khemakhem K, Boudabous J, Cherif L, Ayadi H, Walha A, Moalla Y, et al. Impulsivity in adolescents with major depressive disorder: A comparative tunisian study. Asian J Psychiatr. 2017;28:183-5.

37. Xia C, Wang D, Wu S, Ye J. The development of self-rating idea of suicide scale. Journal of Clinical Psychiatry. 2002;12(2):100-2.

38. Xia C, Wang D, He X, Ye H. Study of self-rating idea undergraduates in the mountain area of southern Zhejiang. Chinese Journal of School Health. 2012;33(2):144-6.

39. Xia C. Self-rating Idea of Suicide Scale, SIOSS. In: Chinese Medical Association. Behavioral medicine inventory manual. Beijing: The Chinese Medicine Electronic Audio and Video Publishing House; 2005. p. 285-7.

40. Zhou L, Xiao S, He X, Li J, Liu H. Reliability and validity of Chinese version of Barratt Impulsiveness Scale- 11 . Chinese Journal of Clinical Psychology. 2006;14(4):343-4.

41. Zung WW. A Self-Rating Depression Scale. Arch Gen Psychiatry. 1965;12:63-70.

42. Zung WW, Richards CB, Short MJ. Self-rating depression scale in an outpatient clinic. Further validation of the SDS. Arch Gen Psychiatry. 1965;13(6):508-15.

43. Colon de Marti LN, Guzman Yunque FS, Guevara-Ramos LM. Early detection of depression using the Zung SelfRating Depression Scale. P R Health Sci J. 1997;16(4):375-9.

44. Kawada T, Suzuki S. Factor structure of self-rating depression scale by Zung and prevalence of depressive state of night shift workers. Sangyo Igaku. 1992;34(2):131-6.

45. Martinez KG, Guiot HM, Casas-Dolz I, Gonzalez-Tejera G, Colon de Marti LN. Applicability of the Spanish Translation of the Zung Self-Rating Depression Scale in a general Puerto Rican population. P R Health Sci J. 2003;22(2):179-85.

46. Wang C, Cai Z, Xu Q. Self-rating Depression Scale: an analysis for 1,340 general participants. Chinese Journal of Nervous and Mental Diseases. 1986;12(5):267-8.

47. IBM Corp. IBM SPSS statistics for windows, version 20.0. Chicago IL: SPSS Inc; 2013.

48. Ringle CM, Wende S, Becker JM. SmartPLS 3. Boenningstedt: SmartPLS GmbH; 2015.

49. Muthen LK, Muthen BO. Mplus 8.3. Los Angeles: StatModel; 2019.

50. Cheong J, Mackinnon DP, Khoo ST. Investigation of Mediational Processes Using Parallel Process Latent Growth Curve Modeling. Structural Equation Modeling A Multidisciplinary Journal. 2003;10(2):238-62. 
51. Soest TV, Hagtvet KA. Mediation Analysis in a Latent Growth Curve Modeling Framework. Structural Equation Modeling A Multidisciplinary Journal. 2011;18(2):289-314.

52. Wen ZL, Hau KT, Marsh HW. STRUCTURAL EQUATION MODEL TESTING: CUTOFF CRITERIA FOR GOODNESS OF FIT INDICES AND CHI-SQUARE TEST. Acta Psychologica Sinica. 2004;36(2):186-94.

53. Bi B, Liu W, Zhou D, Fu X, Qin X, Wu J. Personality traits and suicide attempts with and without psychiatric disorders: analysis of impulsivity and neuroticism. BMC Psychiatry. 2017;17:294.

54. Corruble E, Damy C, Guelfi JD. Impulsivity: a relevant dimension in depression regarding suicide attempts? Journal of Affective Disorders. 1999;53(3):211-5.

55. Dumais A, Lesage AD, Alda M, Rouleau G, Dumont M, Chawky N, et al. Risk factors for suicide completion in major depression: a case-control study of impulsive and aggressive behaviors in men. Am J Psychiatry. 2005;162(11):2116-24.

56. Park CHK, Lee JW, Lee SY, Shim SH, Moon JJ, Paik JW, et al. Implications of Increased Trait Impulsivity on Psychopathology and Experienced Stress in the Victims of Early Trauma With Suicidality. J Nerv Ment Dis. 2018;206(11):840-9.

57. Stanley B, Michel CA, Galfalvy HC, Keilp JG, Rizk MM, Richardson-Vejlgaard R, et al. Suicidal subtypes, stress responsivity and impulsive aggression. Psychiatry Res. 2019;280:112486.

58. Javdani S, Sadeh N, Verona E. Suicidality as a Function of Impulsivity, Callous/Unemotional Traits, and Depressive Symptoms in Youth. other. 2011;120(2):400-13.

59. Bonner RL, Rich AR. Toward a predictive model of suicidal ideation and behavior: some preliminary data in college students. Suicide Life Threat Behav. 1987;17(1):50-63.

60. Conner KR, Duberstein PR, Conwell Y, Seidlitz L, Caine ED. Psychological vulnerability to completed suicide: a review of empirical studies. Suicide and Life-Threatening Behavior. 2015;31(4):367-85.

61. Pennel L, Quesada JL, Dematteis M. Neuroticism and anxious attachment as potential vulnerability factors of repeat suicide attempts. Psychiatry Research. 2018;264(6):46-53.

62. Rich AR, Bonner RL. Concurrent validity of a stress-vulnerability model of suicidal ideation and behavior: a follow-up study. Suicide Life Threat Behav. 1987;17(4):265-70.

\section{Figures}




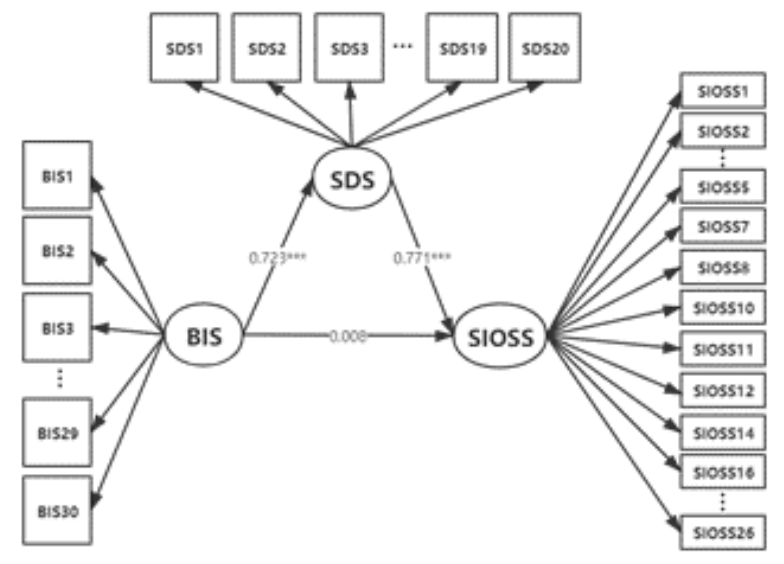

(A)

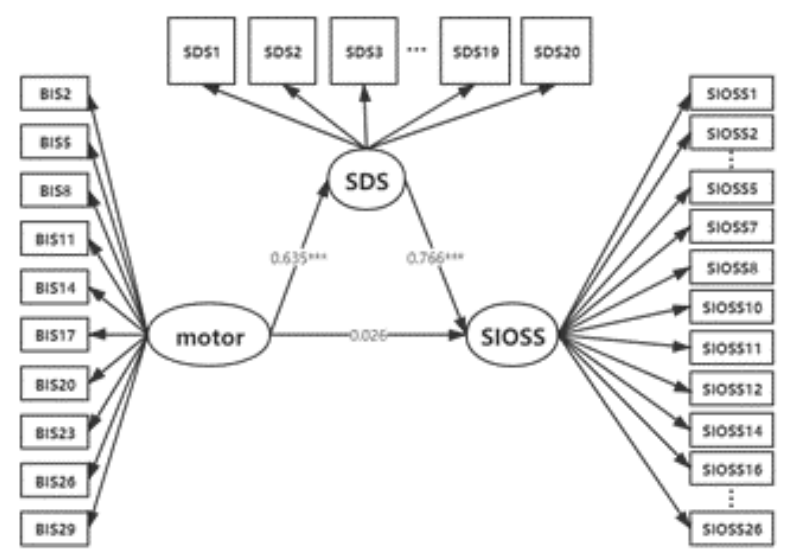

(C)

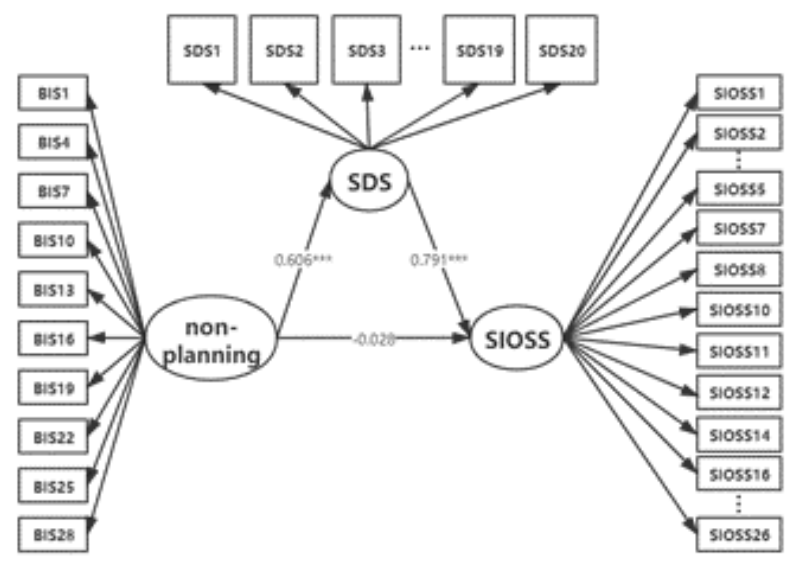

(B)

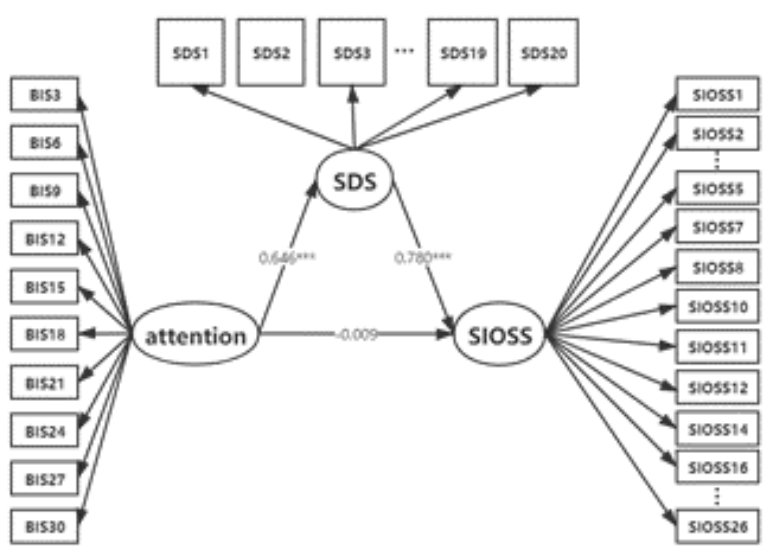

(D)

\section{Figure 1}

Path diagrams of the four mediating models. (A) BIS-SDS-SIOSS, indirect effect coefficient $=0.557, P<0.001$. (B) Nonplanning SDS-SIOSS, indirect effect coefficient $=0.480, P<0.001$. (C) Motor-SDS-SIOSS, indirect effect coefficient $=0.486, P<0.001$. (D) Attention-SDS-SIOSS, indirect effect coefficient $=0.504$. $P<0.001$, $\star \star \star ~ P<0.001$. 


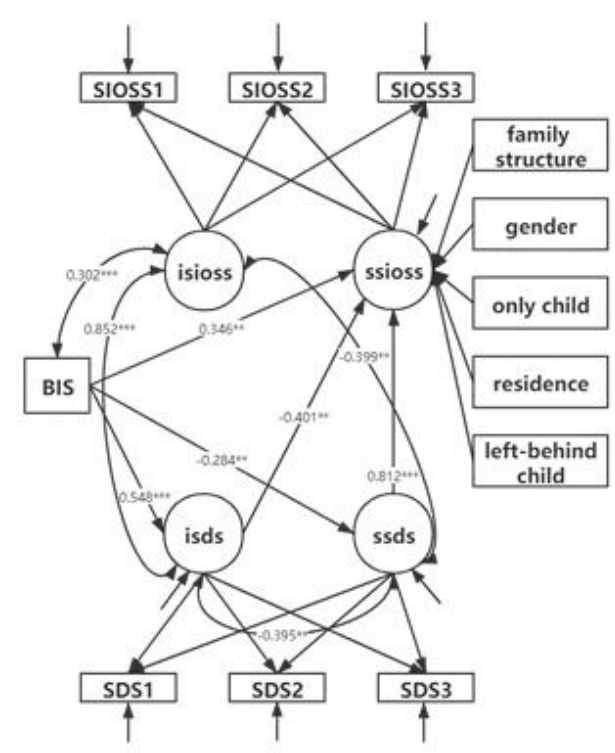

(A)

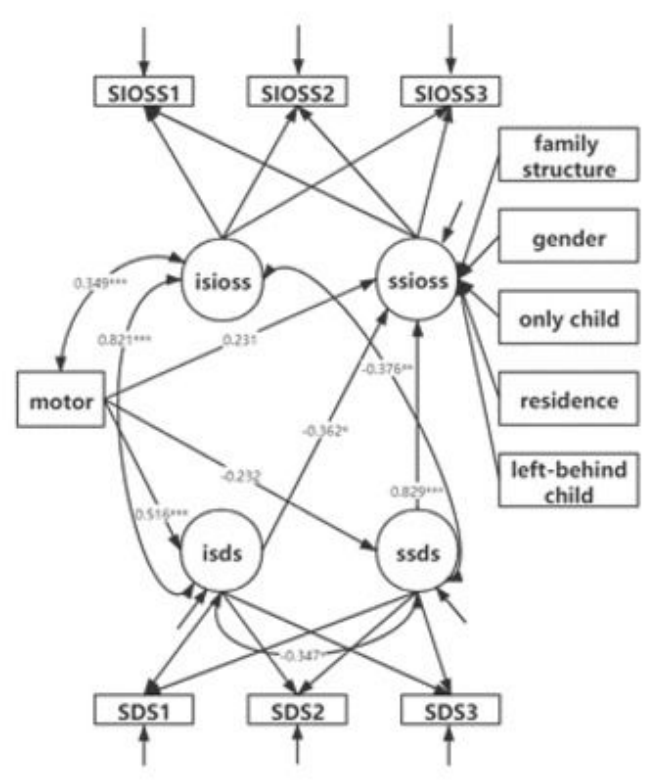

(C)

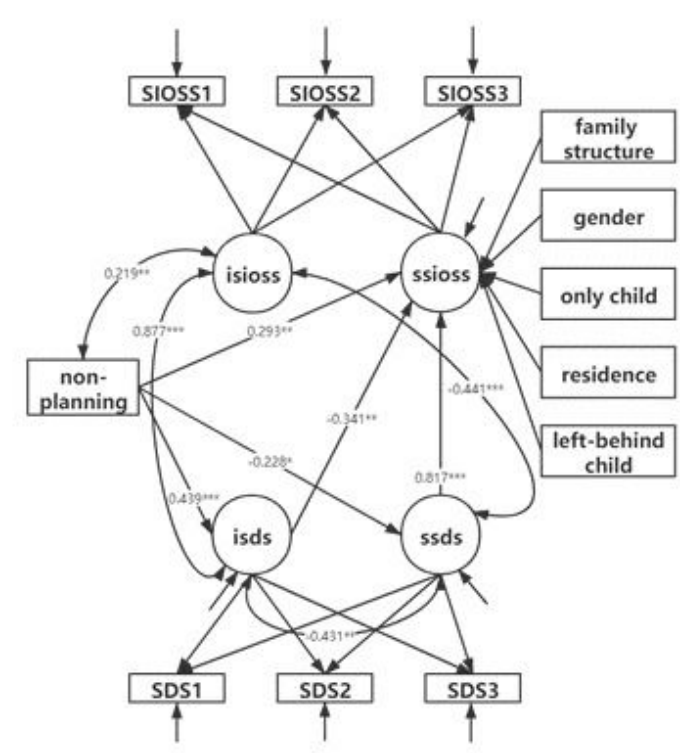

(B)

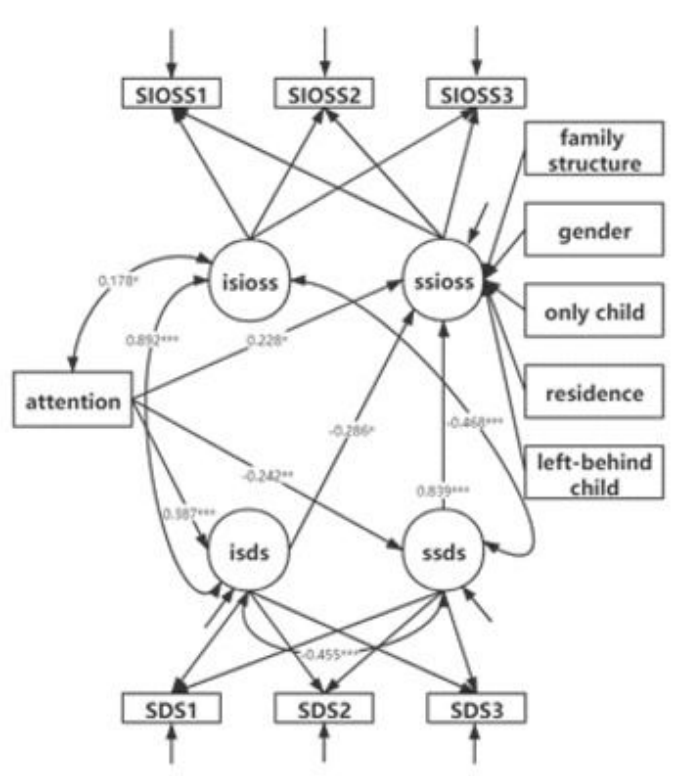

(D)

\section{Figure 2}

Path graphs of four parallel LGCMs. (A) BIS-isds-ssioss indirect effect coefficient=-0.220, P=0.010; BIS-ssds-ssioss indirect effect coefficient $=-0.230, P=0.009$; total indirect effect coefficient $=-0.450, P=0.001$; values: isds $=-0.439$, ssds $=0.079$, isioss $=5.026$, ssioss $=-0.605$. (B) Non-planning-isds-ssioss indirect effect coefficient $=-0.150, P=0.013$; non-planning-ssds-ssioss indirect effect coefficient $=-0.186, P=0.032$; total indirect effect coefficient $=-0.336, P=0.005$; values: isds $=-0.460$, ssds $=0.084$, isioss $=5.024$, ssioss $=-0.605$. (C) Motor-isds-ssioss indirect effect coefficient=-0.187, $\mathrm{P}=0.056$; motor-ssds-ssioss indirect coefficient $=-0.192, \mathrm{P}=0.224$; total indirect effect coefficient $=-0.379, P=0.067$; values: isds $=-0.420$, $s s d s=0.078$, isioss $=5.043$, ssioss $=-0.608$. (D) Attention-isds-ssioss indirect effect coefficient $=-0.111, P=0.035$; attention-ssds-ssioss indirect effect coefficient $=-0.203, P=0.013$; total indirect effect coefficient $=-0.314, P=0.002$; values: isds $=-0.463$, ssds $=0.082$, isioss $=5.029$, $s$ ioss $=-0.607 .{ }^{*} P<0.05$, 
${ }^{\star *} \mathrm{P}<0.01,{ }^{\star} * * \mathrm{P}<0.001$. SIOSS1, SIOSS2, and SIOSS3: SIOSS scores at Wave0, Wave1, and Wave2; SDS1, SDS2, and SDS3: SDS scores at Wave0, Wave1, and Wave2 